\title{
AN X-RAY SURVEY OF OB ASSOCIATIONS IN THE LARGE MAGELLANIC CLOUD
}

\author{
QINGDE WANG AND DAVID J. HELFAND \\ Columbia Astrophysics Laboratory \\ 538 West 120th Street \\ New York, NY 10027
}

\begin{abstract}
Based on Einstein IPC data, we have completed a census of X-ray emission from and around OB associations in the LMC. In addition to an apparent correlation of young, $\mathrm{X}$-ray bright supernova remnants with recent star formation regions, we detect diffuse X-ray emission from over two dozen other associations; luminosities in the 0.16 $3.5 \mathrm{keV}$ band range from $\sim 3 \times 10^{34}$ (the detection threshold) to $\sim 10^{36} \mathrm{erg}^{-1}$. The mean $\mathrm{X}$-ray luminosity of the $\sim 50$ undetected associations is $\sim 10^{34} \mathrm{erg}^{-1}$ and the emission from all associations contributes $\sim 4 \%$ to the total diffuse $X$-ray emission from the galaxy. Implications of this survey for ISM bubble evolution is discussed.
\end{abstract}

In a recent paper by Wang et al. (1990) we have summarized previous work on Einstein observations of the LMC, presented a revised discrete X-ray source catalog based on all available IPC images, and described a series of calibration and contaminationremoval algorithms necessary for the study of diffuse X-ray emission with the IPC. In a series of papers (Wang and Helfand 1990a,b,c), we have presented detailed studies of bright $X$-ray emission regions: the 30 Doradus Nebula and the supergiant bubble LMC 2, as well as a general study of the $\mathrm{X}$-ray properties of the $\mathrm{OB}$ associations. The following is a brief description of our X-ray survey of the diffuse X-ray emission from the OB associations.

Among 86 associations covered in the survey, 22 discrete $\mathrm{X}$-ray sources are found which fall within the boundaries of $17 \mathrm{OB}$ associations. Among these are six $\mathrm{OB}$ associations LH 53, 75, 83, 88, 90 and 99, each of which apparently contains one of the $28 \mathrm{X}$-ray emitting SNRs in the LMC (also see Chu and Kennicutt 1988). The expected chance coincidence rate is $<1$. It will be interesting to study these remnants in detail to determine how their location in or near the low-density cavities created by their parent association will affect their evolution.

Significant diffuse X-ray emission is detected from the vicinities of LH39, 47, 54, 60, 63, $76,81,87,90$, and 104. Their X-ray fluxes fall in a narrow range of $.01-0.05 I P C$ ct s$^{-1}$, corresponding to X-ray luminosities of $\sim 10^{35}$ to $\sim 10^{36} \mathrm{erg} \mathrm{s}^{-1}$. All of these associations are relatively compact, presumably young clusters with diameters $<90 \mathrm{pc}$ and a median stellar content of 30, somewhat higher than the average of $\sim 20$ stars per association for the sample as a whole. 
Emission from the associations such as LH47, 54, and 90 has been resolved; they exhibit shell-like X-ray morphologies which generally follow the $\mathrm{H} \alpha$-emitting gas. These $\mathrm{X}$-ray sources are both larger and fainter than those identified as SNRs, and show a rough correlation between source intensity and the spectral hardness of the emission.

In an attempt to find lower surface brightness, more extended X-ray emission in the vicinity of $\mathrm{OB}$ associations, we searched the remaining 69 associations with two larger source-searching apertures. Maintaining our $4 \sigma$ detection threshold as used in our $2^{\prime} .5$ aperture discrete source search (Wang et al. 1990), we find six additional detections with a $6^{\prime}$ aperture and fifteen with an $8^{\prime}$ aperture.

The integrated X-ray count rate from $8^{\prime}$-diameter circles centered on each of these $69 \mathrm{OB}$ associations is $0.33 \mathrm{ct} \mathrm{s}^{-1}$. The integrated emission from the detected discrete source associations excluding the SNRs, a foreground star, and LMC X-1 is $0.32 \mathrm{ct} \mathrm{s}^{-1}$, providing an upper limit (owing to possible chance coincidences) to the total contribution of individual $\mathrm{OB}$ associations to the diffuse X-rays from the Cloud: $\lesssim 0.65 \mathrm{ct} \mathrm{s}^{-1}$ or $\sim 4 \%$ of the total diffuse emission. For the remaining (undetected) 53 associations, we find an average flux of $\sim 2 \times 10^{-3} \mathrm{ct} \mathrm{s}^{-1}$ per association corresponding to a mean luminosity of $\sim 2 \times 10^{34} \mathrm{erg} \mathrm{s}^{-1}$. This compares with an expected stellar contribution from 2009 stars per cluster of $\sim 5 \times 10^{32} \mathrm{erg} \mathrm{s}^{-1}$.

As we have compiled the first census of the $\mathrm{X}$-ray properties of $\mathrm{OB}$ associations in the Cloud, we have begun to combine the X-ray information with observations at other wavelength bands in order to explore the evolution of giant bubbles.

We have calculated a composite $X$-ray spectrum of a wind-driven bubble and shown that very soft $\mathrm{X}$-rays emitted from the conductive layer at the edge of the bubble dominate the bubble's cooling; this low energy radiation is almost entirely absorbed on the way to the Earth. The expected X-ray flux in the IPC broad band is typically below the observed values by a factor larger than 10. The two brightest bubbles, N44(LH47) and N157C(LH90), also have the hardest X-ray spectra observed; a fit of their combined spectral data to thermal plasma models (Raymond et al. 1977) results in a temperature range of $\sim 0.8$ to $1.8 \mathrm{keV}$ (with a $90 \%$ significance level). Their high X-ray luminosities and hard $\mathrm{X}$-ray spectra require either wind-driven bubbles evolving in a dense medium $\left(\gtrsim 10^{2} \mathrm{~cm}^{-3}\right)$ or SNR-filled-bubbles. Most of the other association detections also appear to require Xray emission enhanced over the level predicted by simple bubble models. SNRs hitting the dense shells of young (typically several $10^{6}$ years old) bubbles may produce such enhanced $\mathrm{X}$-ray emission.

This work was supported under a grant from NASA NAG8-664.

\section{REFERENCES}

Chu, Y.-H., and Kennicutt, R. C. Jr. 1988, Astron. J., 95, 1874.

Raymond, J.C., and Smith, B.W. 1977, Ap.J. Suppl., 35, 419.

Wang, Q. and Helfand, D. J. 1990a,b,c Ap.J. (submitted).

Wang, Q., Hamilton, T., Helfand, D. J., and Wu, X-Y 1990, Ap.J., (in press). 